\title{
Platelet Count to Spleen Diameter Ratio for the Diagnosis of Gastroesophageal Varices in Liver Cirrhosis: A Systematic Review and Meta-Analysis
}

\author{
Runhua Chen, ${ }^{1}$ Han Deng, ${ }^{2}$ Xia Ding, ${ }^{3}$ Chune Xie, ${ }^{4}$ Wei Wang, ${ }^{5}$ and Qian Shen ${ }^{6}$ \\ ${ }^{1}$ Department of Gastroenterology, Dongfang Hospital, Beijing University of Chinese Medicine, Beijing, China \\ ${ }^{2}$ Postgraduate College, Dalian Medical University, Dalian, China \\ ${ }^{3}$ Department of Hospital Administration, Beijing University of Chinese Medicine, Beijing, China \\ ${ }^{4}$ Department of Personnel, Dongfang Hospital, Beijing University of Chinese Medicine, Beijing, China \\ ${ }^{5}$ Department of Cardiology, Wangjing Hospital, China Academy of Chinese Medical Sciences, Beijing, China \\ ${ }^{6}$ Department of Massage and Physiotherapy, Dongfang Hospital, Beijing University of Chinese Medicine, Beijing, China
}

Correspondence should be addressed to Qian Shen; rbd144@163.com

Received 3 September 2016; Accepted 30 November 2016; Published 8 February 2017

Academic Editor: Nianping Feng

Copyright (C) 2017 Runhua Chen et al. This is an open access article distributed under the Creative Commons Attribution License, which permits unrestricted use, distribution, and reproduction in any medium, provided the original work is properly cited.

Platelet count to spleen diameter ratio (PSR) was studied extensively as a noninvasive method of diagnosis for varices. The present study aimed to systematically assess the performance of PSR in the diagnosis of varices. PubMed, EMBASE, and article references were searched. The summary receiver operating characteristic curves (AUSROCs), sensitivities, specificities, positive and negative likelihood ratio, and diagnostic odds ratio were calculated. The heterogeneity, quality, and publication bias of studies were evaluated. Subgroup and sensitivity analyses were performed. A total of 49 papers were included. The AUSROCs of PSR for any varices and high-risk varices were 0.8719 and 0.8132 , respectively. The summary sensitivities of PSR for any varices and high-risk varices were 0.84 and 0.78 , respectively. The summary specificities of PSR for any varices and high-risk varices were 0.78 and 0.67 , respectively. The AUSROC of PSR for any varices at the threshold of 909 was 0.8867 . The AUSROC of PSR for any varices in viral liver cirrhosis was 0.8675 . The overall quality of studies was moderate. Significant heterogeneity and publication bias existed in the study. In conclusion, PSR can be used to identify varices in liver cirrhosis. PSR had a high sensitivity in viral liver cirrhosis.

\section{Introduction}

Gastroesophageal varices are one of the major complications of liver cirrhosis. Early detection of varices in cirrhotic patients is crucial to ensure timely initiation of prophylactic therapies. Platelet count to spleen diameter ratio (PSR) was first proposed by Giannini et al. to predict the presence of varices in 2003 [1]. An increasing number of studies have since evaluated the accuracy of PSR in the detection of varices, albeit drawing inconsistent conclusions. In two previous meta-analyses regarding the diagnostic accuracy of PSR in predicting the presence of varices, Ying et al. [2] recommended using PSR to identify varices to decrease the use of upper gastrointestinal endoscopy, while Chawla et al. [3] found that PSR has low grade evidence to replace upper gastrointestinal endoscopy as a noninvasive method for varices. The performance of PSR for varices is still not unified at present, which has limited the use of PSR in clinical practice. Thus, we conducted this systematic review and meta-analysis to evaluate the performance of PSR for varices.

\section{Methods}

Selection, data extraction, and quality assessment of studies were conducted by two investigators (RC and QS) independently. Disagreement between the two investigators was resolved by a consensus.

2.1. Search Strategy and Selection Criteria. PubMed and EMBASE were searched on May 27, 2016. The search terms were as follows: $((()(($ platelet count to spleen diameter ratio) 
OR PSR) OR PC/SD)) OR ((((platelet) OR platelet count $))$ AND ((spleen) OR spleen diameter)))) AND liver cirrhosis) AND varices. Relevant references were also screened. Duplicates, commentaries, reviews, case reports, letters, metaanalyses, book sections, and meeting abstracts were excluded. The inclusion criteria were as follows: (1) participants should be diagnosed with liver cirrhosis; (2) upper gastrointestinal endoscopy should be performed as the reference tests for the diagnosis of varices; (3) PSR should be performed as alternative tests for the diagnosis of varices; (4) diagnostic accuracy data of PSR on the diagnosis of varices were available. The language and publication year were not limited.

2.2. Data Extraction. The following data from each study was extracted: the first author, publication year, region, study design, total number of patients, age, sex, etiology of liver cirrhosis, hepatocellular carcinoma (HCC), Child-Pugh class, location of varices (i.e., esophageal varices $[\mathrm{EV}]$ and/or gastric varices $[\mathrm{GV}])$, prevalence of any varices and/or highrisk (or large) varices, cut-off value, true positive (TP) value, false positive (FP) value, false negative (FN) value, and true negative (TN) value. Missing values were calculated using the following formulae: sensitivity $=\mathrm{TP} /(\mathrm{TP}+\mathrm{FN})$, and specificity $=\mathrm{TN} /(\mathrm{TN}+\mathrm{FP})$. The raw TP, FN, FP, and TN numbers of included studies were shown in Supplementary Table 1 (see Supplementary Material available online at https://doi.org/10.1155/2017/7407506).

2.3. Quality Assessment. The quality of each study was assessed by QUADAS-2 (Quality Assessment of Diagnostic Accuracy Studies-2) tool [4]. This tool comprises four domains: patient selection, index test, reference standard, and flow and timing. The risk of bias in each domain was rated as "low risk," "high risk," and "unclear risk" with signaling questions. The applicability concerns in the first three domains were assessed as "low concern," "high concern," and "unclear concern." If the total number of "low risk" and "high concern" was equal or greater than 6 in a study, the study was considered as high quality.

2.4. Statistical Analysis. The area under the summary receiver operating characteristic curves (AUSROCs) with standard errors (SEs) and $Q$ indexes with SEs, summary sensitivities and specificities with $95 \%$ confidence intervals (CIs), summary positive and negative likelihood ratios (PLRs and NLRs) with 95\% CIs, and summary diagnostic odds ratios (DORs) with $95 \%$ CIs were calculated using statistical software (MetaDisc software version 1.4). We analyzed these data using the random-effects model. The diagnostic threshold was analyzed by Spearman correlation coefficient and $p$ value. $p<$ 0.05 showed a statistically significant diagnostic threshold effect. Therefore, only AUSROCs with SEs and Q indexes with SEs were calculated. The heterogeneity among studies was evaluated by Chi-square test and inconsistency index. A statistically significant heterogeneity was defined as $p<0.1$ and/or $I^{2}>50 \%$.

To explore the publication bias, we performed Deeks' funnel plot asymmetry test in Stata 12.0 (College Station, TX,
USA). Sensitivity analyses were performed via removing each study to evaluate the impact on the pooled results of the removed study.

We calculated the diagnostic accuracy of PSR for various cut-off values in predicting the presence of any and highrisk varices. If there were multiple different cut-off values in the same paper, we selected the optimal cut-off values. PSR for any varices at threshold of 909 was the most generally accepted cut-off value at present. Thus, we performed subgroup analyses using the cut-off value of 909. In addition, we performed subgroup analyses based on the etiology of cirrhosis, region, study design, prevalence of varices, sample size, and study quality for any varices.

\section{Results}

3.1. Selection of Studies. A total of 345 papers were selected from PubMed $(n=120)$, EMBASE $(n=218)$, and manual search $(n=7)$. The flow diagram of study selection was shown in Figure 1. 112 duplicates and 145 irrelevant papers were excluded. Then, we screened 88 full-text articles for eligibility. 39 papers which lacked relevant diagnostic data were excluded. Among them, 28 papers were applied only with abstracts. Finally, 49 papers $[1,5-52]$ were included in our study. 42 and 16 papers were about any varices and highrisk varices, respectively.

3.2. Characteristics of Studies. The characteristics of studies were shown in Table 1. Our meta-analysis included a total of 6274 patients. 22 papers were prospective studies. 2 papers $[10,20]$ were published in abstracts and 47 papers were fulltexts. The etiologies of cirrhosis were alcohol, schistosomiasis, and viral hepatitis in $1[12], 2[5,32]$, and $13[9,19,22,26$, $27,30,33,36,39,40,42-44]$ papers, respectively. Two papers were about $\mathrm{EV}$ and $\mathrm{GV}[8,12]$, and the rest of the papers were about EV alone. In two papers, all patients had Child-Pugh A $[22,40]$. The diagnostic accuracy of PSR for any varices at threshold of 909 was reported in 19 papers.

3.3. Quality Assessment of Studies. The overall quality of the included studies was not very high (Supplementary Table 2). In the patient selection domain, only 17 papers were rated as "low risk." In the index test and reference standard domains, 10 and 14 papers were rated as "low risk." Most papers had not reported whether investigators were blinded when interpreting the results of index test and reference standard. 2 papers were rated as "high risk" in flow and timing domain as the interval time between index test and reference standard was greater than 3 months. 44 papers have "high concern" in patient selection domain. In addition, all papers were rated as "high concern" in index test and reference standard domains. 11 papers were considered with high quality.

3.4. Overall Results. Significant threshold effect was not found in overall meta-analyses.

Diagnostic accuracy of PSR for the presence of any varices was pooled from 42 papers $[1,5-10,13,14,16-19,21-34$, 36-40, 42-50, 52]. The AUSROC was 0.8719 (Figure 2(a)). The summary sensitivity and specificity were $0.84(95 \%$ 


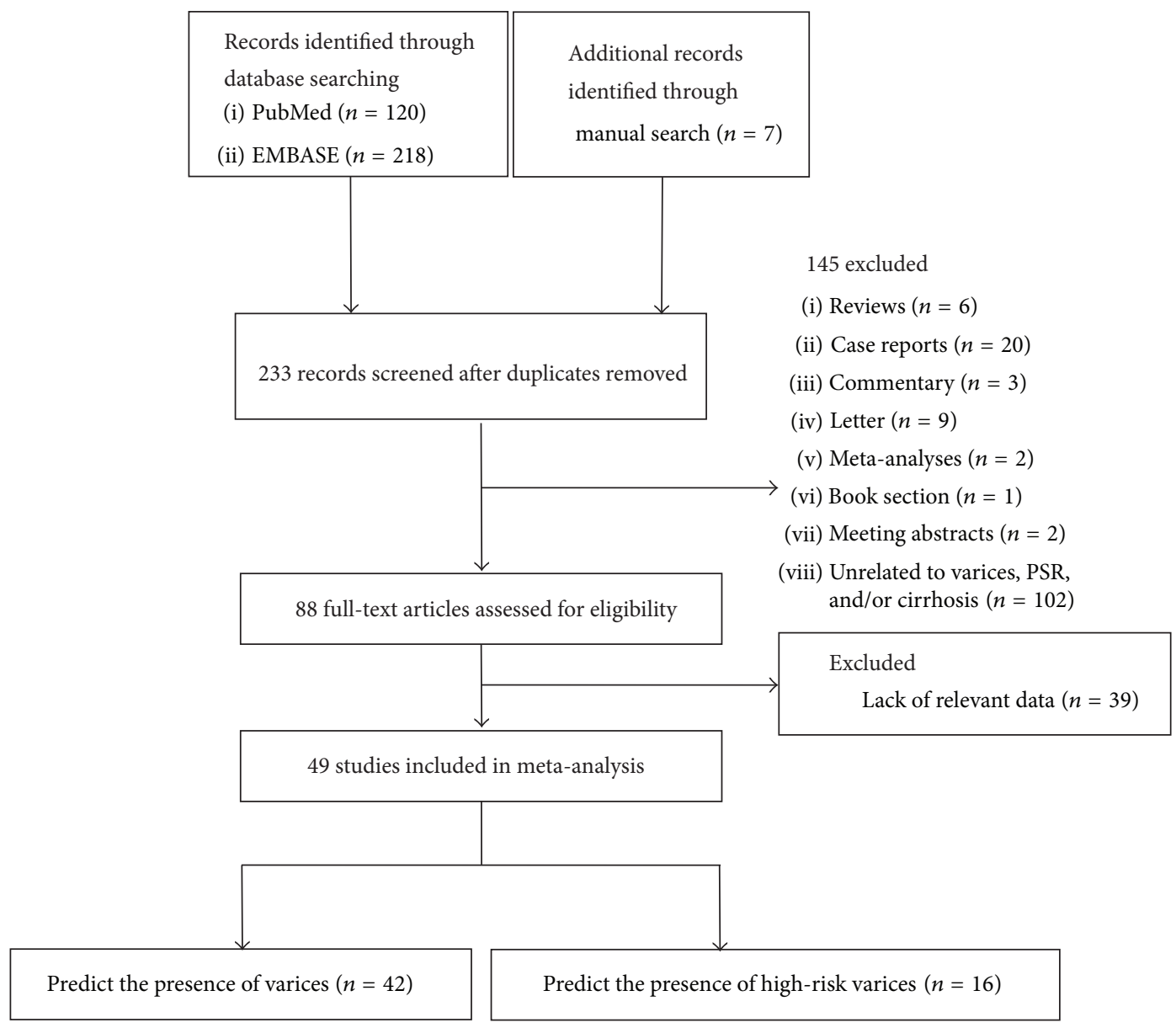

FIGURE 1: Flowchart of study selection.

CI: $0.83-0.85)$ and 0.78 (95\% CI: $0.76-0.79)$, respectively (Figure 3). The summary PLR, NLR, and DOR were 3.54 (95\% CI: $2.75-4.56), 0.17$ (95\% CI: 0.12-0.23), and 25.32 (95\% CI: 15.72-40.77), respectively.

Diagnostic accuracy of PSR for the presence of high-risk varices was pooled from 16 papers $[6,7,11-13,15,17,19,20,22$, $25,29,30,35,41,51]$. The AUSROC was 0.8132 (Figure 2(b)). The summary sensitivity and specificity were 0.78 (95\% CI: $0.75-0.81$ ) and 0.67 (95\% CI: 0.64-0.71), respectively (Figure 4). The summary PLR, NLR, and DOR were 2.54 (95\% CI: 1.99-3.24), 0.32 (95\% CI: 0.24-0.44), and 9.08 (95\% CI: 5.33-15.47), respectively. The diagnostic accuracy of PSR for high-risk varices was lower than PSR for any varices.

3.5. Subgroup Results. The subgroup results were summarized in Table 2. Significant threshold effect was found in the subgroup of South America. Thus, their diagnostic accuracy was not combined.

3.6. Heterogeneity. Significant heterogeneity between papers was found in most analyses except for the subgroup of North America and sample size less than 100.

3.7. Sensitivity Analyses. Sensitivity analysis results were similar to the overall meta-analysis results. The heterogeneity remained significant (data not shown).
3.8. Publication Bias. The publication bias existed in the study $(p=0.007)$.

\section{Discussions}

In our study, the AUSROC of PSR for any varices was 0.8719 . The summary sensitivity and specificity were 0.84 and 0.78 , respectively. The diagnostic accuracy of PSR for high-risk varices was lower than PSR for any varices. The diagnostic accuracy of PSR for varices at threshold of 909 was similar to PSR at various thresholds. While the summary sensitivity (0.92) in viral liver cirrhosis was improved over that of mixed etiologies, they had the same summary specificities (0.78). The subgroup analysis of Asia had the highest AUSROC (0.9195).

The high diagnostic accuracy of PSR for varices can be explained as follows. Varices and hypersplenism are the results of portal hypertension. The platelet count can be influenced by many factors in cirrhotic patients other than hypersplenism. The decreased thrombopoietin production is the reason. Thrombopoietin is mainly produced by hepatocytes and the quantity can be largely reduced when the hepatocytes was damaged. In addition, the shortened platelet mean lifetime and myelotoxic effects of alcohol or hepatitis viruses also reduced the platelet count. Splenomegaly is the clinical manifestation of hypersplenism. Thus, a combined 


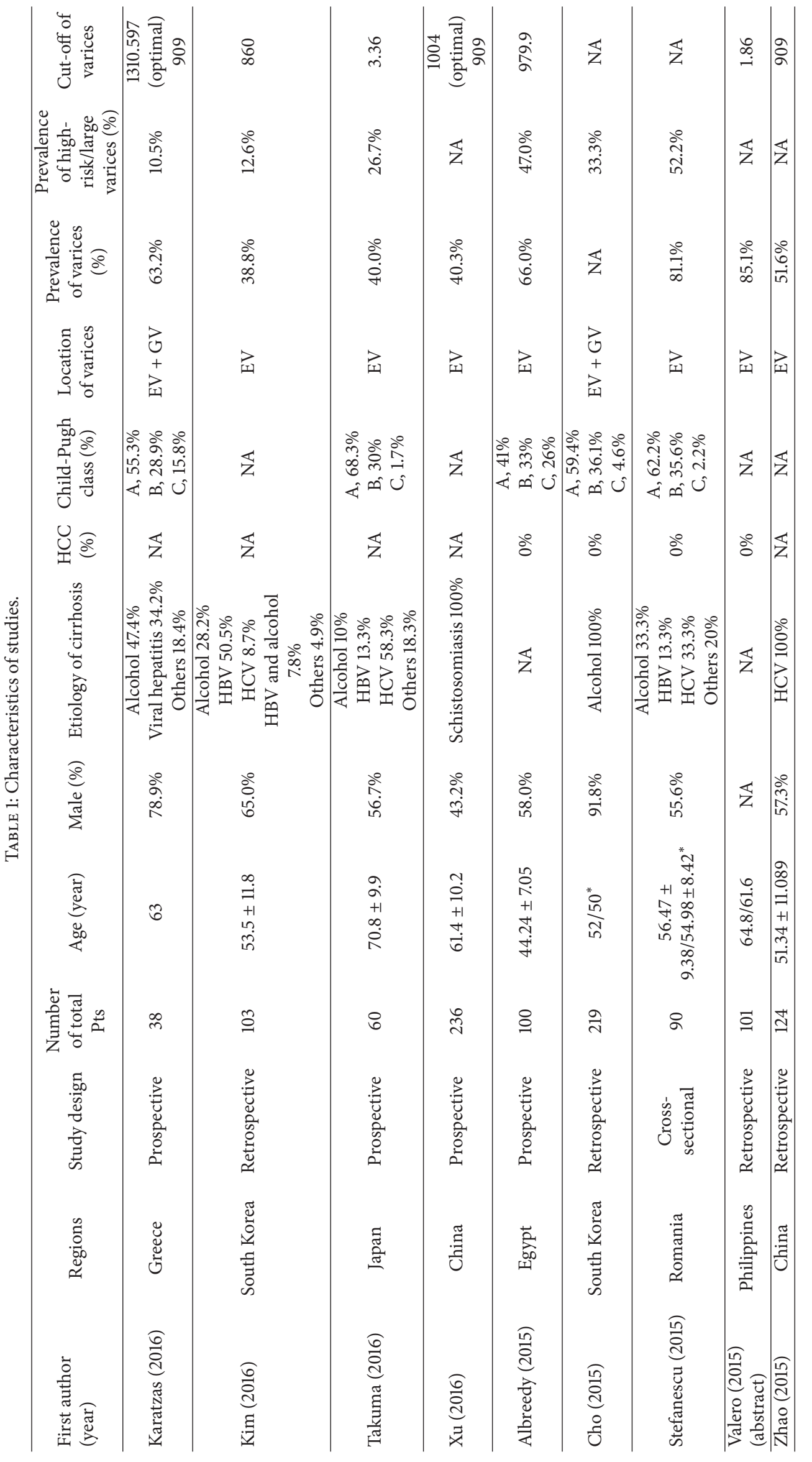




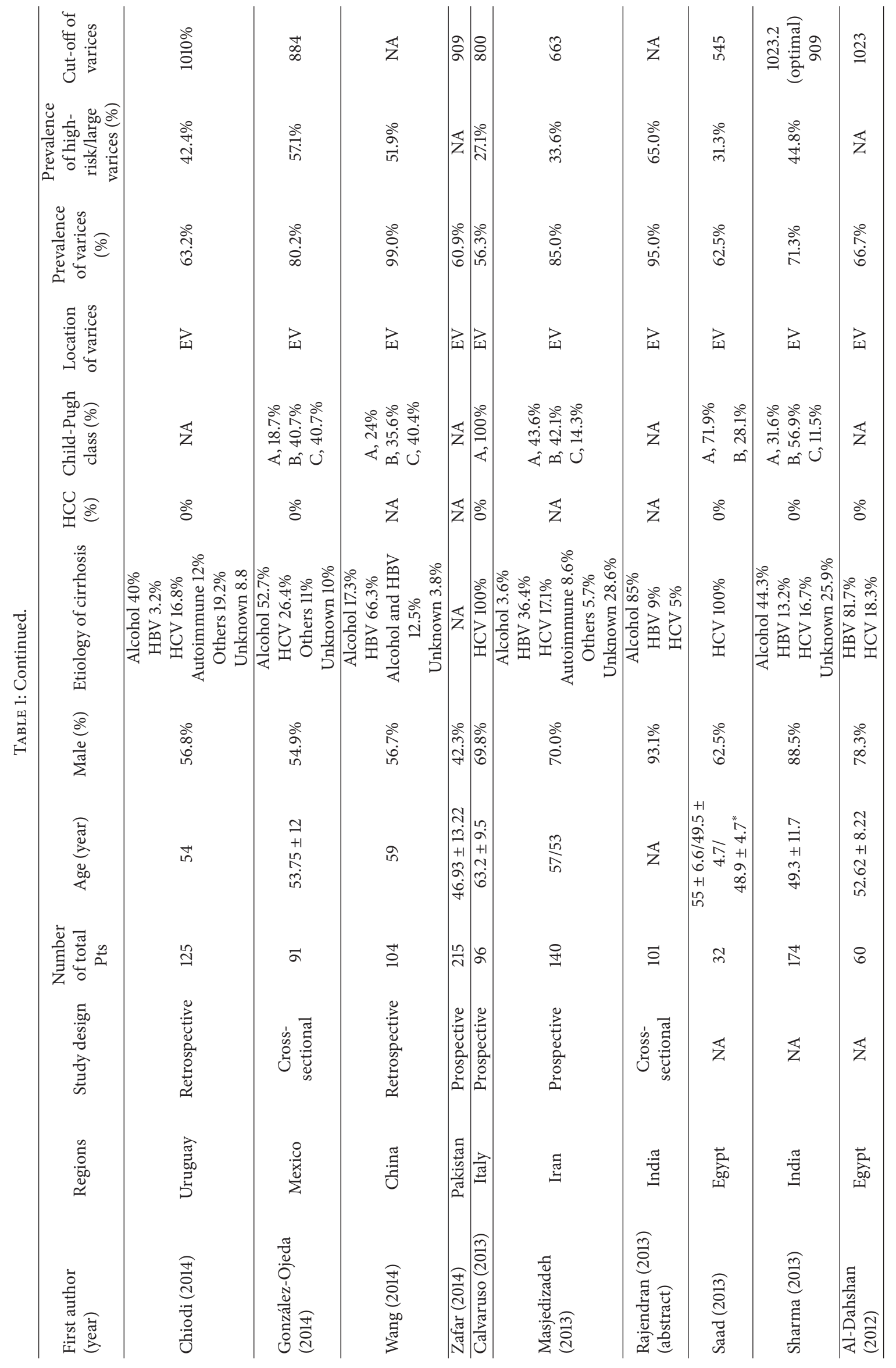




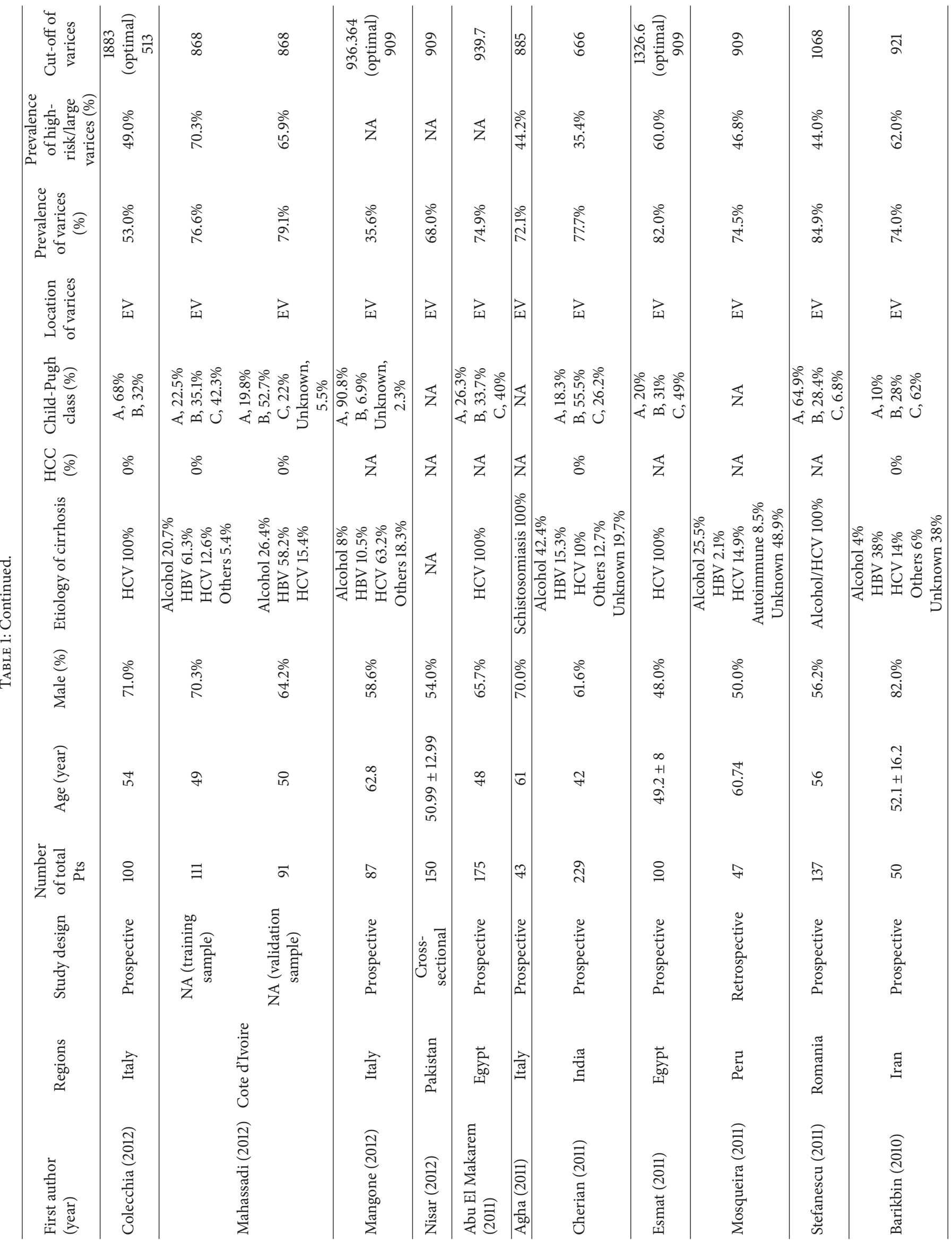




\begin{tabular}{|c|c|c|c|c|c|c|c|c|c|}
\hline 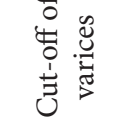 & ڤे & స్రి & 送 & ڤे & वे & $\overleftrightarrow{z}$ & $\tilde{\Omega}$ & సે & 蒙言高 \\
\hline 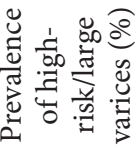 & $\begin{array}{l}\text { oे } \\
\text { oे } \\
\text { के }\end{array}$ & 艺 & $\frac{\stackrel{\partial}{\circ}}{\stackrel{\infty}{+}}$ & $\begin{array}{l}\stackrel{\circ}{\circ} \\
\stackrel{\infty}{\oplus}\end{array}$ & $\begin{array}{l}\text { बे } \\
\text { త్ }\end{array}$ & $\begin{array}{l}\text { ळे } \\
\text { वें }\end{array}$ & $\overleftrightarrow{z}$ & $\overleftrightarrow{z}$ & 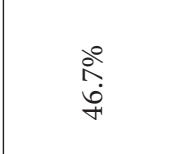 \\
\hline 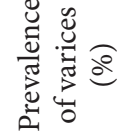 & $\stackrel{\text { ஸे }}{\text { ָे }}$ & $\begin{array}{l}\text { वे } \\
\text { को } \\
\infty\end{array}$ & $\begin{array}{l}\stackrel{0}{0} \\
\stackrel{i}{i}\end{array}$ & $\begin{array}{l}\text { in } \\
\text { in } \\
\text { in }\end{array}$ & $\begin{array}{l}\text { ओें } \\
\text { भे }\end{array}$ & 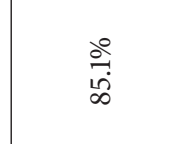 & $\begin{array}{l}0 \\
\dot{0} \\
\dot{0} \\
0\end{array}$ & $\begin{array}{l}\text { oे } \\
\text { iे }\end{array}$ & $\begin{array}{l}\stackrel{2}{2} \\
\stackrel{2}{R}\end{array}$ \\
\hline 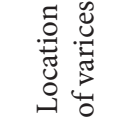 & 㞌 & 觅 & 学 & 贫 & 贫 & 学 & 空 & 空 & 空 \\
\hline 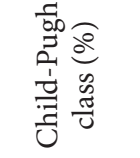 & 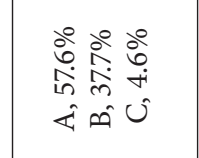 & 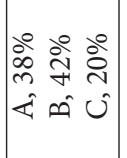 & $\overleftrightarrow{z}$ & $\overleftrightarrow{z}$ & 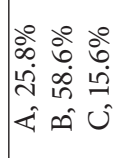 & 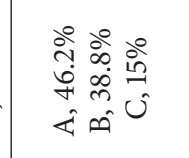 & 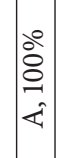 & $\overleftrightarrow{z}$ & 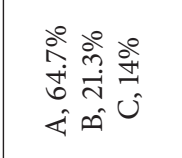 \\
\hline 总 & $\overleftrightarrow{z}$ & $\overleftrightarrow{z}$ & 8̊ & $\overleftrightarrow{Z}$ & $\therefore$ & $\overleftrightarrow{z}$ & $\therefore$ & $\overleftrightarrow{z}$ & $\overleftrightarrow{z}$ \\
\hline 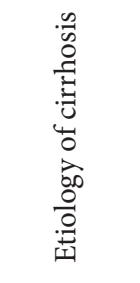 & 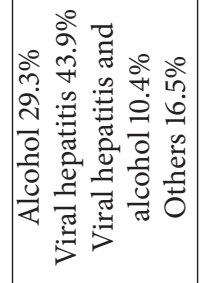 & 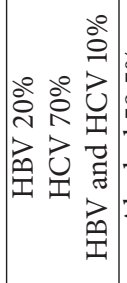 & 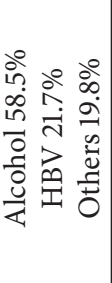 & 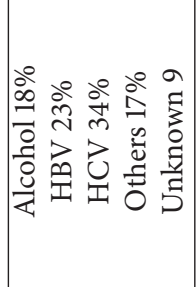 & 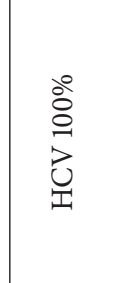 & 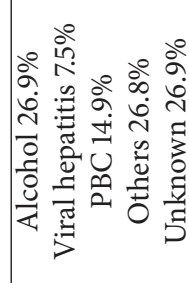 & 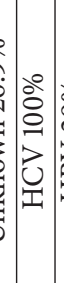 & 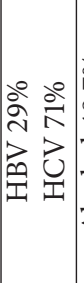 & 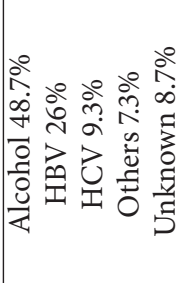 \\
\hline 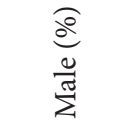 & $\begin{array}{l}\stackrel{\circ}{2} \\
\text { Lे } \\
\text { in }\end{array}$ & $\begin{array}{l}\stackrel{\circ}{0} \\
\stackrel{+}{+}\end{array}$ & $\begin{array}{l}\stackrel{2}{ } \\
\text { ते }\end{array}$ & $\begin{array}{l}\text { oे } \\
\text { ti }\end{array}$ & $\begin{array}{l}\dot{0} \\
\text { iे } \\
\text { in }\end{array}$ & $\begin{array}{c}\stackrel{े}{े} \\
\stackrel{F}{F}\end{array}$ & $\mid \begin{array}{l}0 \\
\grave{\lambda} \\
\text { in }\end{array}$ & \begin{tabular}{|l} 
oे \\
i̊
\end{tabular} & $\begin{array}{l}\text { ठे } \\
\text { +ं }\end{array}$ \\
\hline 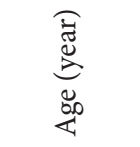 & $\begin{array}{l}0 \\
\dot{1} \\
+1 \\
0 \\
b \\
\dot{0} \\
i\end{array}$ & $\begin{array}{l}\infty \\
\infty \\
\infty \\
+1 \\
\dot{0} \\
\dot{q}\end{array}$ & \& & ட & Fे & $\begin{array}{l}\underset{1}{3} \\
+1 \\
+1 \\
0\end{array}$ & $\mid \begin{array}{c}1 \\
2 \\
+1 \\
+1 \\
+1 \\
0 \\
\end{array}$ & $\overleftrightarrow{z}$ & $\vec{n}$ \\
\hline 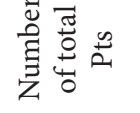 & ప్ర & in & $\stackrel{8}{\circ}$ & $\hat{n}$ & $\bar{m}$ & $\hat{\sigma}$ & $\stackrel{+}{\stackrel{\Delta}{O}}$ & 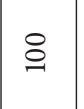 & $\stackrel{\circ}{n}$ \\
\hline 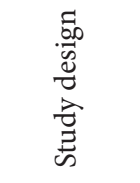 & $\overleftrightarrow{z}$ & $\overleftrightarrow{z}$ & 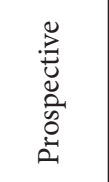 & 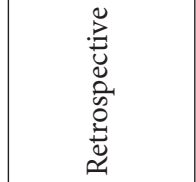 & 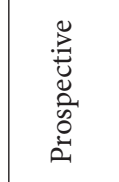 & 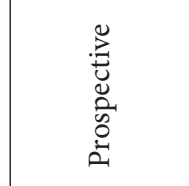 & 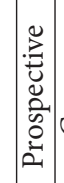 & 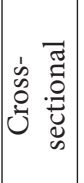 & 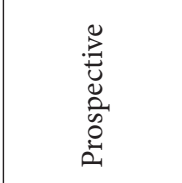 \\
\hline 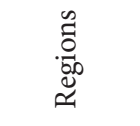 & 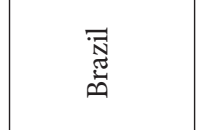 & 营 & ت্ّ & $\tilde{D}$ & 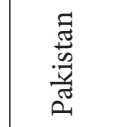 & 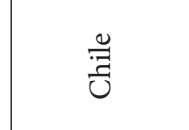 & 폴 & 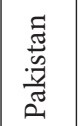 & ت্ّ \\
\hline 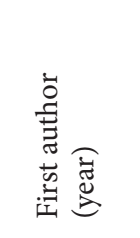 & 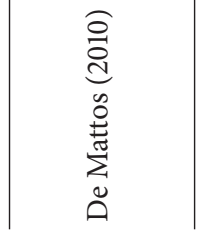 & 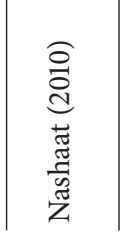 & 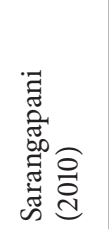 & 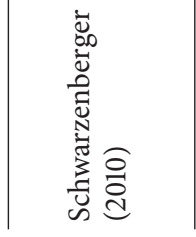 & 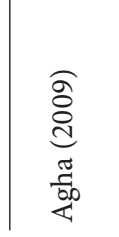 & 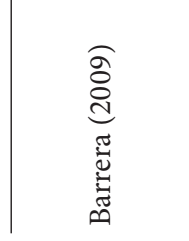 & 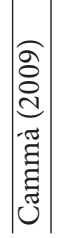 & 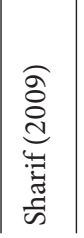 & 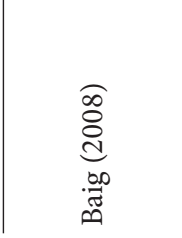 \\
\hline
\end{tabular}




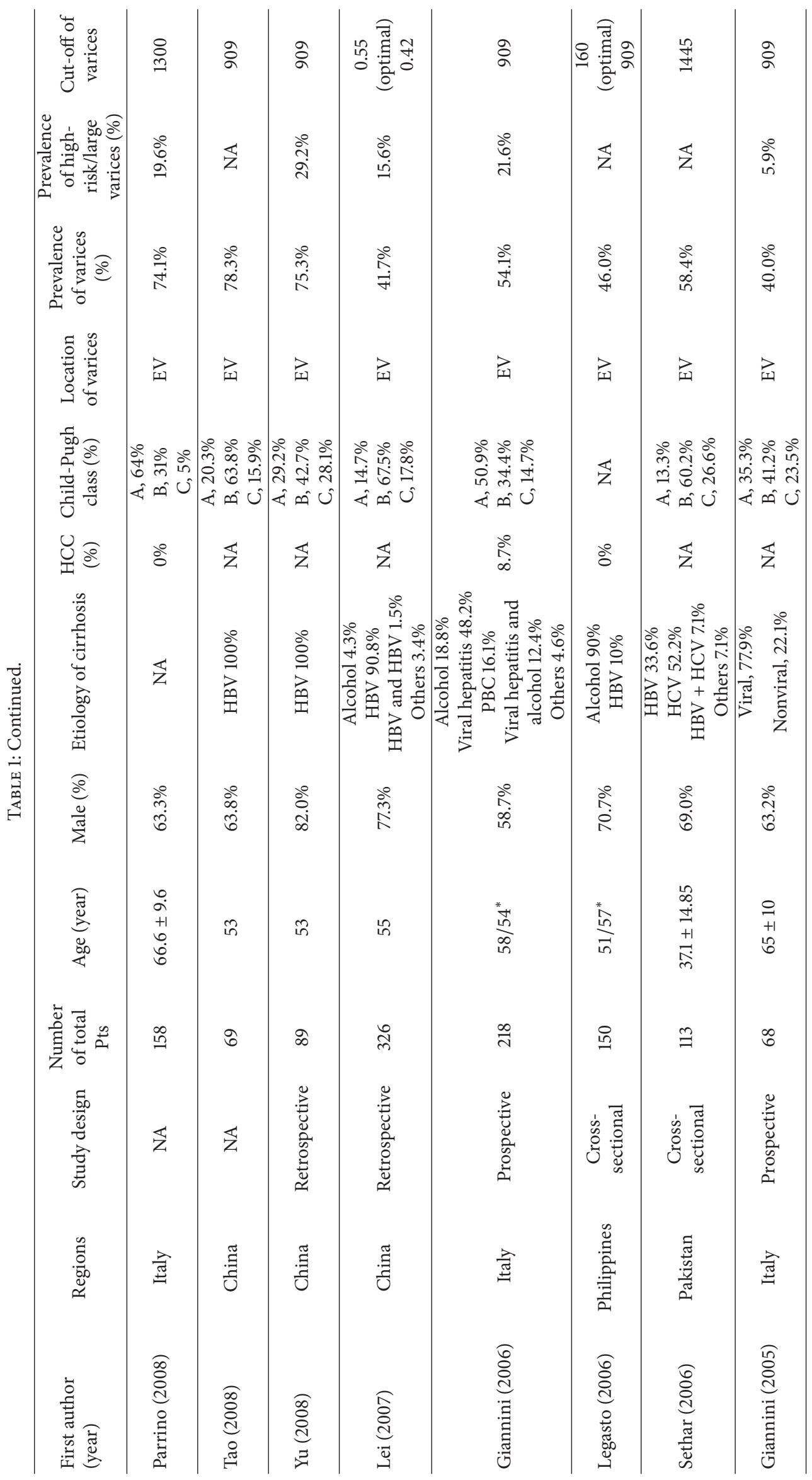




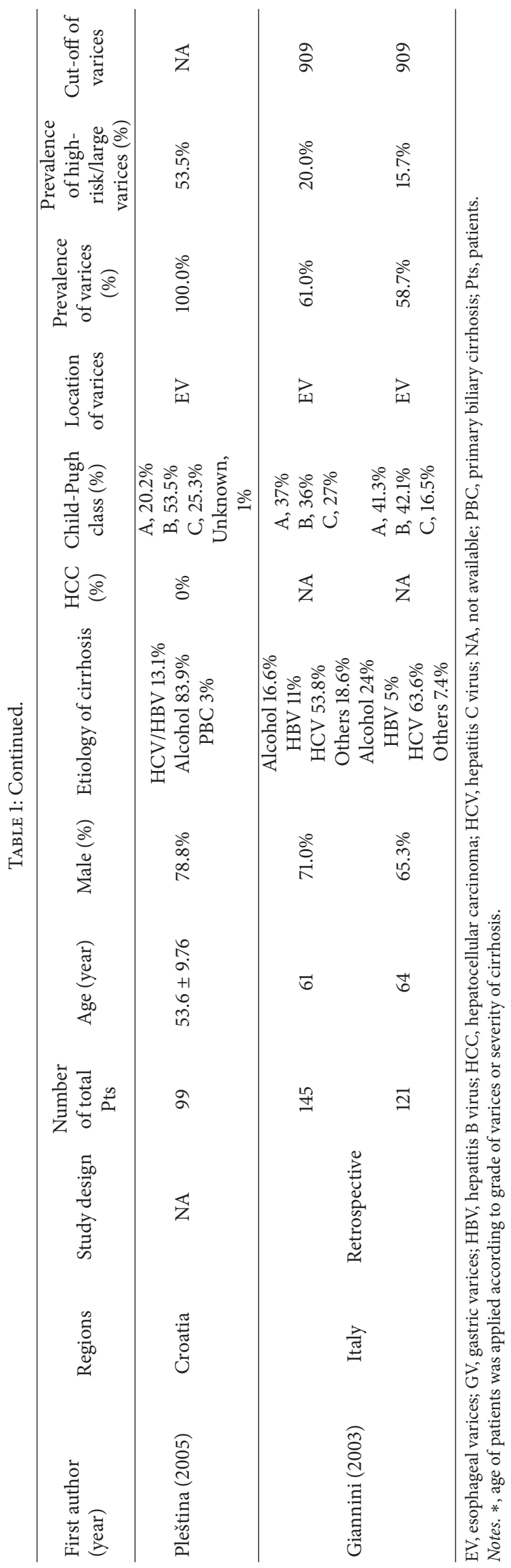


TABLE 2: Results of meta-analyses in subgroups for any varices.

\begin{tabular}{lcccccc}
\hline Groups & AUSROC & Sensitivity (95\% CI) & Specificity (95\% CI) & PLR (95\% CI) & NLR (95\% CI) & DOR (95\% CI) \\
\hline Threshold of 909 & 0.8867 & $0.84(0.82-0.86)$ & $0.80(0.78-0.82)$ & $3.95(2.66-5.86)$ & $0.21(0.13-0.32)$ & $25.06(11.84-53.03)$ \\
Patients with viral hepatitis & 0.8675 & $0.92(0.90-0.94)$ & $0.78(0.74-0.81)$ & $3.80(2.04-7.08)$ & $0.11(0.06-0.23)$ & $37.76(14.43-98.84)$ \\
High quality studies & 0.876 & $0.84(0.81-0.87)$ & $0.77(0.74-0.8)$ & $3.64(2.11-6.3)$ & $0.15(0.07-0.33)$ & $23.79(10.35-54.7)$ \\
Prospective studies & 0.8748 & $0.86(0.84-0.88)$ & $0.76(0.73-0.79)$ & $3.59(2.39-5.39)$ & $0.12(0.07-0.22)$ & $33.85(15.67-73.15)$ \\
Region & & & & & & \\
$\quad$ Europe & 0.8289 & $0.83(0.8-0.86)$ & $0.65(0.61-0.7)$ & $2.58(1.81-3.69)$ & $0.2(0.11-0.38)$ & $15.46(7.07-33.79)$ \\
$\quad$ Asia & 0.9195 & $0.86(0.84-0.87)$ & $0.86(0.84-0.88)$ & $5.18(3.5-7.65)$ & $0.11(0.06-0.2)$ & $55.48(24.27-126.81)$ \\
$\quad$ Africa & 0.8537 & $0.87(0.84-0.9)$ & $0.71(0.64-0.77)$ & $3.28(1.74-6.16)$ & $0.16(0.08-0.34)$ & $23.31(9.2-59.09)$ \\
$\quad$ North America & NA & $0.82(0.75-0.88)$ & $0.67(0.56-0.77)$ & $2.45(1.76-3.4)$ & $0.27(0.18-0.39)$ & $9.06(4.73-17.36)$ \\
Sample size & & & & & & \\
$\quad<100$ & 0.7895 & $0.81(0.77-0.84)$ & $0.7(0.65-0.75)$ & $2.57(2.01-3.3)$ & $0.25(0.15-0.4)$ & $12.58(6.34-24.97)$ \\
$\quad \geq 100$ & 0.9012 & $0.85(0.83-0.86)$ & $0.79(0.77-0.81)$ & $4.1(2.92-5.77)$ & $0.14(0.09-0.22)$ & $34.51(18.84-63.2)$ \\
Prevalence of varices & & & & & & \\
$\quad<50 \%$ & 0.8804 & $0.91(0.88-0.93)$ & $0.86(0.83-0.88)$ & $5.29(3.03-9.23)$ & $0.11(0.04-0.3)$ & $54.16(14.29-205.25)$ \\
$\quad \geq 50 \%$ & 0.8633 & $0.83(0.81-0.84)$ & $0.73(0.7-0.75)$ & $3.15(2.45-4.05)$ & $0.19(0.13-0.26)$ & $21.12(12.85-34.71)$ \\
\hline
\end{tabular}

AUSROC, area under the summary receiver operating characteristic curves; CI, confidence interval; DOR, diagnostic odds ratios; NLR, negative likelihood ratio; PLR, positive likelihood ratio.

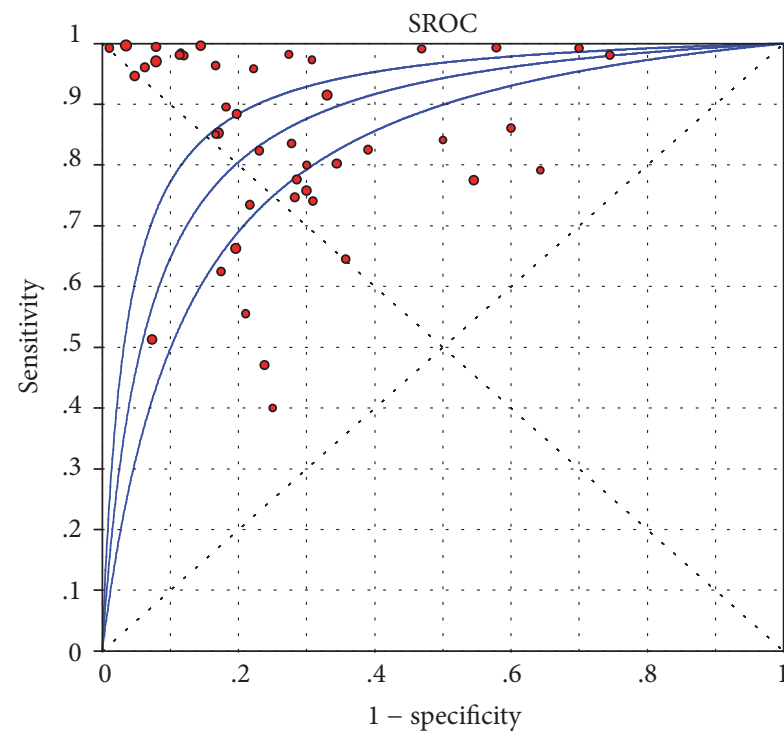

Symmetric SROC

$\mathrm{AUC}=0.8719$

$\mathrm{SE}(\mathrm{AUC})=0.0244$

$Q^{*}=0.8024$

$\operatorname{SE}\left(Q^{*}\right)=0.0242$

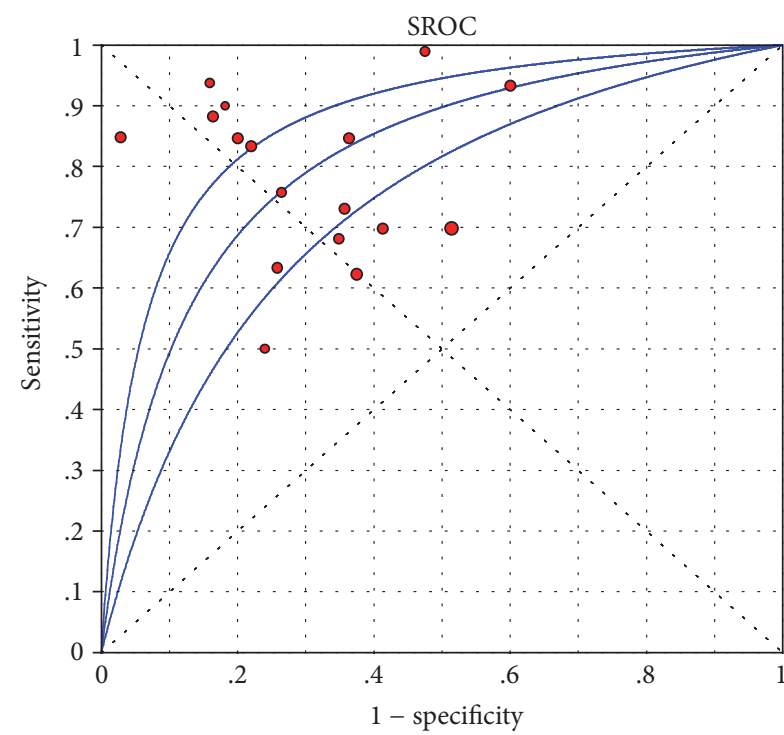

Symmetric SROC

AUC $=0.8132$

$\mathrm{SE}(\mathrm{AUC})=0.0337$

$Q^{*}=0.7475$

$\operatorname{SE}\left(Q^{*}\right)=0.0300$

(a)

(b)

FIgURE 2: AUSROCs of PSR for varices in liver cirrhosis. (a) Any size varices; (b) high-risk varices.

index of platelet count and spleen diameter has much more relevance with portal hypertension and varices than the sole decreased platelet count [1].

In clinic practice, the measure of spleen diameter and platelet count is easily obtainable during the routine ultrasonography and serum examination. PSR is convenient, cheap, and noninvasive. Based on our study, we recommend that those patients whose PSR is less than 909 should undergo upper gastrointestinal endoscopy to evaluate the grade of varices. $80 \%$ of patients whose value of PSR is greater than 909 can avoid unnecessary upper gastrointestinal endoscopic examination.

Compared with the two previous studies, our metaanalysis included a greater number of studies without limiting the publication language and cut-off values. Compared with other noninvasive methods, PSR has an upper-middle 


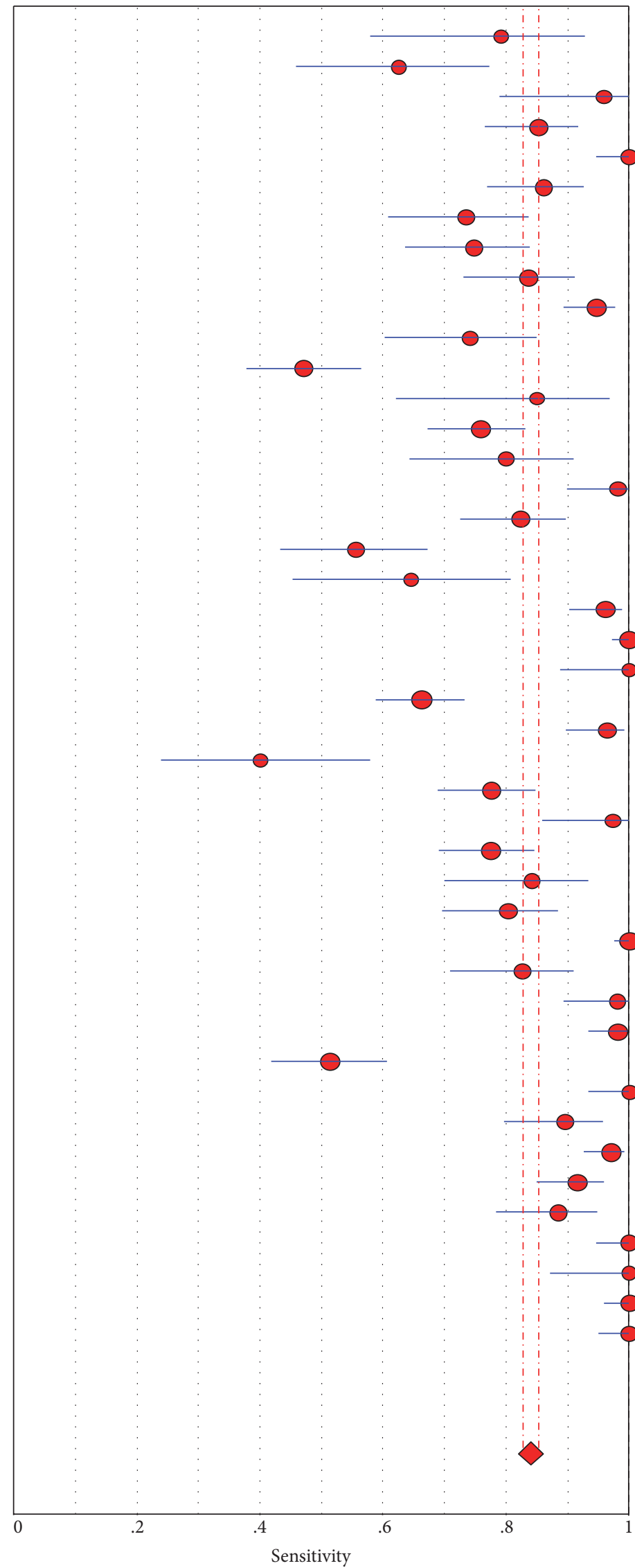

Karatzas et al. (2016)

Kim et al. (2016)

Takuma et al. (2016)

Xu et al. (2016)

Albreedy (2015)

Valero and Olympia (2015)

Zhao et al. (2015)

Chiodi et al. (2014)

González-Ojeda et al. (2014)

Zafar and Latif (2014)

Calvaruso et al. (2013)

Masjedizadeh et al. (2013)

Saad et al. (2013)

Sharma et al. (2013)

Al-Dahshan (2012)

Colecchia et al. (2012)

Mahassadi et al. (2012) a

Mahassadi et al. (2012) b

Mangone et al. (2012)

Nisar et al. (2012)

Abu El Makarem et al. (2011)

Agha et al. (2011)

Cherian et al. (2011)

Esmat and Rashid (2011)

Mosqueira et al. (2011)

Stefanescu et al. (2011)

Barikbin et al. (2010)

De Mattos et al. (2010)

Nashaat et al. (2010)

Schwarzenberger et al. (2010)

Agha et al. (2009)

Cammà et al. (2009)

Sharif et al. (2009)

Baig et al. (2008)

Parrino et al. (2008)

Tao et al. (2008)

Yu et al. (2008)

Lei and Huang (2007)

Giannini et al. (2006)

Legasto et al. (2006)

Sethar et al. (2006)

Giannini et al. (2005)

Giannini et al. (2003) a

Giannini et al. (2003) b

Pooled sensitivity $=0.84$ (0.83 to 0.85 )

$\chi^{2}=671.49 ; \mathrm{df}=43(p=0.0000)$

Inconsistency $\left(I^{2}\right)=93.6 \%$

$$
\begin{gathered}
\text { Sensitivity }(95 \% \text { CI) } \\
0.79(0.58-0.93) \\
0.63(0.46-0.77) \\
0.96(0.79-1.00) \\
0.85(0.77-0.92) \\
1.00(0.95-1.00) \\
0.86(0.77-0.93) \\
0.73(0.61-0.84) \\
0.75(0.64-0.84) \\
0.84(0.73-0.91) \\
0.95(0.89-0.98) \\
0.74(0.60-0.85) \\
0.47(0.38-0.56) \\
0.85(0.62-0.97) \\
0.76(0.67-0.83) \\
0.80(0.64-0.91) \\
0.98(0.90-1.00) \\
0.82(0.73-0.90) \\
0.56(0.43-0.67) \\
0.65(0.45-0.81) \\
0.96(0.90-0.99) \\
1.00(0.97-1.00) \\
1.00(0.89-1.00) \\
0.66(0.59-0.73) \\
0.96(0.90-0.99) \\
0.40(0.24-0.58) \\
0.78(0.69-0.85) \\
0.97(0.86-1.00) \\
0.78(0.69-0.85) \\
0.84(0.70-0.93) \\
0.80(0.70-0.89) \\
1.00(0.98-1.00) \\
0.83(0.71-0.91) \\
0.98(0.89-1.00) \\
0.98(0.93-1.00) \\
0.51(0.42-0.61) \\
1.00(0.93-1.00) \\
0.90(0.80-0.96) \\
0.97-0.93-0.99) \\
\end{gathered}
$$

(a)

FIgure 3: Continued. 


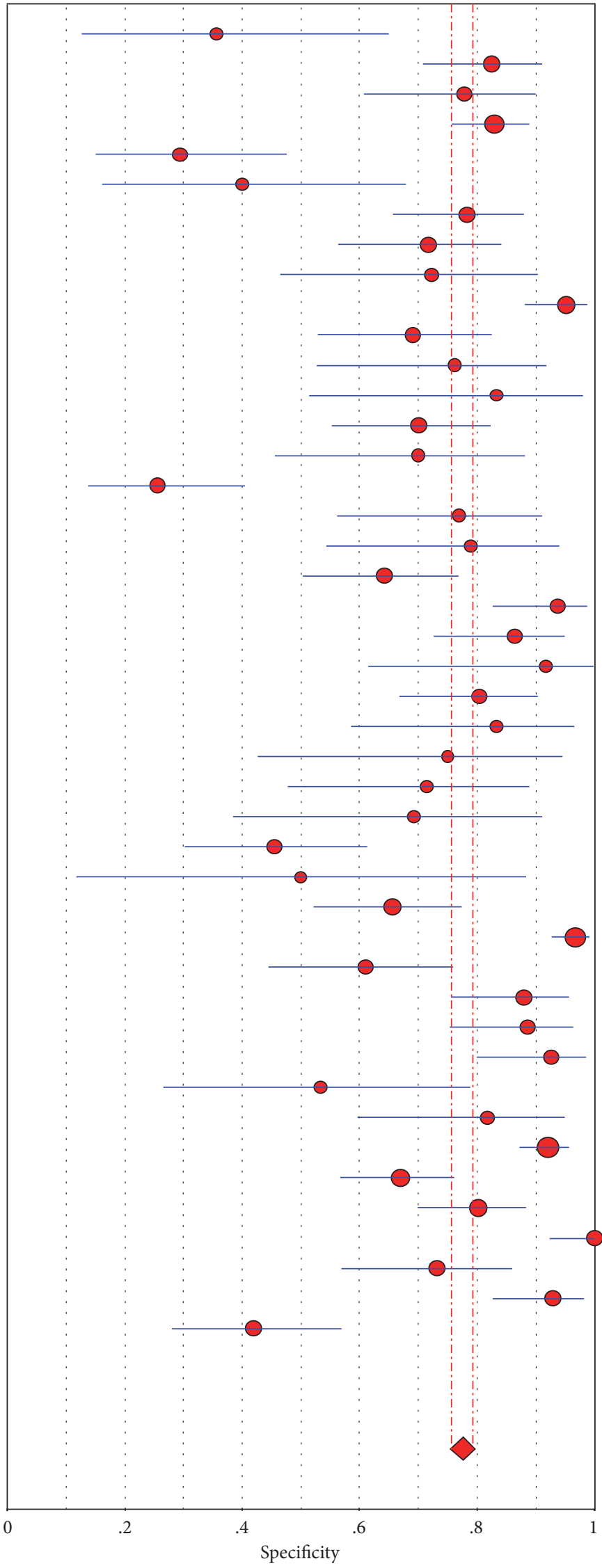

Karatzas et al. (2016)

Kim et al. (2016)

Takuma et al. (2016)

Xu et al. (2016)

Albreedy (2015)

Valero and Olympia (2015)

Zhao et al. (2015)

Chiodi et al. (2014)

González-Ojeda et al. (2014)

Zafar and Latif (2014)

Calvaruso et al. (2013)

Masjedizadeh et al. (2013)

Saad et al. (2013)

Sharma et al. (2013)

Al-Dahshan (2012)

Colecchia et al. (2012)

Mahassadi et al. (2012) a

Mahassadi et al. (2012) b

Mangone et al. (2012)

Nisar et al. (2012)

Abu El Makarem et al. (2011)

Agha et al. (2011)

Cherian et al. (2011)

Esmat and Rashid (2011)

Mosqueira et al. (2011)

Stefanescu et al. (2011)

Barikbin et al. (2010)

DeMattos et al. (2010)

Nashaat et al. (2010)

Schwarzenberger et al. (2010)

Agha et al. (2009)

Cammà et al. (2009)

Sharif et al. (2009)

Baig et al. (2008)

Parrino et al. (2008)

Tao et al. (2008)

Yu et al. (2008)

Lei and Huang (2007)

Giannini et al. (2006)

Legasto et al. (2006)

Sethar et al. (2006)

Giannini et al. (2005)

Giannini et al. (2003) a

Giannini et al. (2003) b
Specificity (95\% CI)

$0.36(0.13-0.65)$

$0.83(0.71-0.91)$

$0.78(0.61-0.90)$

$0.83(0.76-0.89)$

$0.29(0.15-0.47)$

$0.40(0.16-0.68)$

$0.78(0.66-0.88)$

$0.72(0.57-0.84)$

$0.72(0.47-0.90)$

$0.95(0.88-0.99)$

$0.69(0.53-0.82)$

$0.76(0.53-0.92)$

$0.83(0.52-0.98)$

$0.70(0.55-0.82)$

$0.70(0.46-0.88)$

$0.26(0.14-0.40)$

$0.77(0.56-0.91)$

$0.79(0.54-0.94)$

$0.64(0.50-0.77)$

$0.94(0.83-0.99)$

$0.86(0.73-0.95)$

$0.92(0.62-1.00)$

$0.80(0.67-0.90)$

$0.83(0.59-0.96)$

$0.75(0.43-0.95)$

$0.71(0.48-0.89)$

$0.69(0.39-0.91)$

$0.45(0.30-0.61)$

$0.50(0.12-0.88)$

$0.66(0.52-0.77)$

$0.97(0.93-0.99)$

$0.61(0.45-0.76)$

$0.88(0.76-0.95)$

$0.89(0.75-0.96)$

$0.93(0.80-0.98)$

$0.53(0.27-0.79)$

$0.82(0.60-0.95)$

$0.92(0.87-0.96)$

$0.67(0.57-0.76)$

$0.80(0.70-0.88)$

$1.00(0.92-1.00)$

$0.73(0.57-0.86)$

$0.93(0.83-0.98)$

$0.42(0.28-0.57)$

Pooled specificity $=0.78$ (0.76 to 0.79 )

$\chi^{2}=361.20 ; \mathrm{df}=43(p=0.0000)$

Inconsistency $\left(I^{2}\right)=88.1 \%$ 


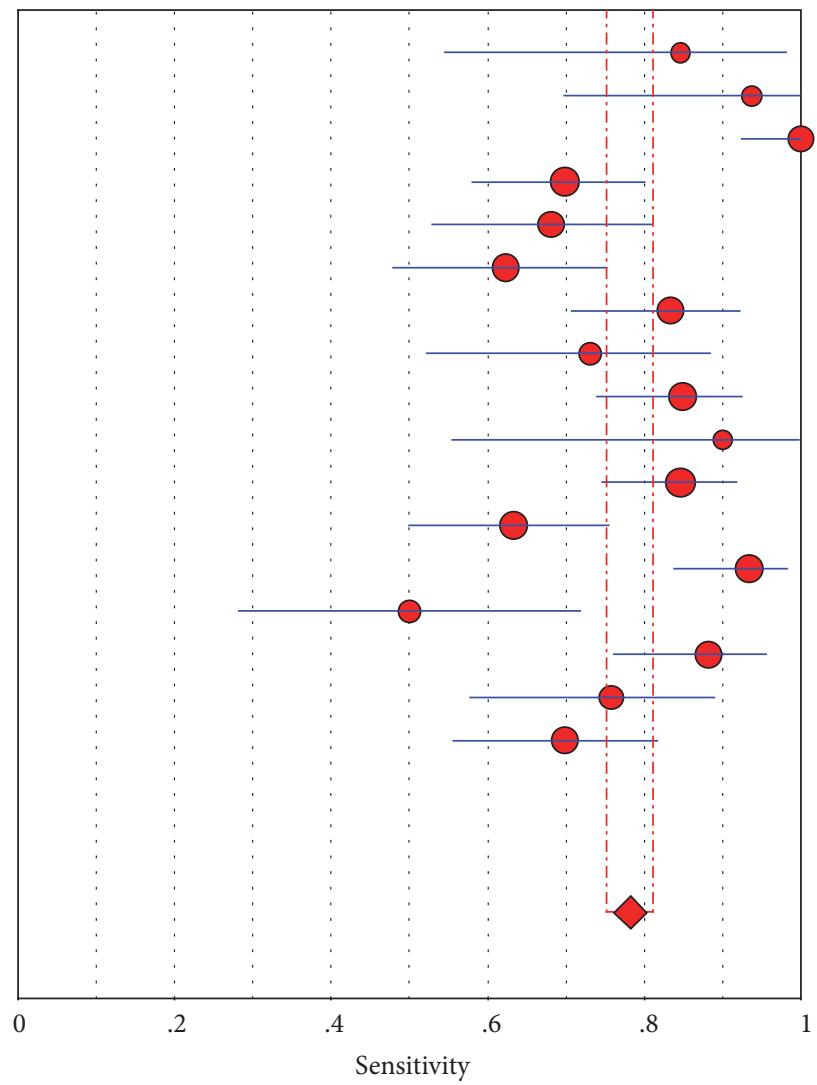

Kim et al. (2016)

Takuma et al. (2016)

Albreedy (2015)

Cho et al. (2015)

Stefanescu et al. (2015)

Chiodi et al. (2014)

Wang et al. (2014)

Calvaruso et al. (2013)

Rajendran et al. (2013)

Saad et al. (2013)

Mahassadi et al. (2012) a

Mahassadi et al. (2012) b

Esmat and Rashid (2011)

Mosqueira et al. (2011)

Sarangapani et al. (2010)

Barrera et al. (2009)

Pleština et al. (2005)

Pooled sensitivity $=0.78$ (0.75 to 0.81 )

$\chi^{2}=75.98 ; \mathrm{df}=16(p=0.0000)$

Inconsistency $\left(I^{2}\right)=78.9 \%$

Kim et al. (2016)

Takuma et al. (2016)

Albreedy (2015)

Cho et al. (2015)

Stefanescu et al. (2015)

Chiodi et al. (2014)

Wang et al. (2014)

Calvaruso et al. (2013)

Rajendran et al. (2013)

Saad et al. (2013)

Mahassadi et al. (2012) a

Mahassadi et al. (2012) b

Esmat and Rashid (2011)

Mosqueira et al. (2011)

Sarangapani et al. (2010)

Barrera et al. (2009)

Pleština et al. (2005)
Sensitivity (95\% CI)

$0.85(0.55-0.98)$

$0.94(0.70-1.00)$

$1.00(0.92-1.00)$

$0.70(0.58-0.80)$

$0.68(0.53-0.81)$

$0.62(0.48-0.75)$

$0.83(0.71-0.92)$

$0.73(0.52-0.88)$

$0.85(0.74-0.92)$

$0.90(0.55-1.00)$

$0.85(0.75-0.92)$

$0.63(0.50-0.75)$

$0.93(0.84-0.98)$

$0.50(0.28-0.72)$

$0.88(0.76-0.96)$

$0.76(0.58-0.89)$

$0.70(0.56-0.82)$
Specificity (95\% CI)

$0.80(0.70-0.88)$

$0.84(0.70-0.93)$

$0.53(0.29-0.76)$

$0.49(0.40-0.57)$

$0.65(0.49-0.79)$

$0.63(0.50-0.74)$

$0.78(0.64-0.88)$

$0.64(0.52-0.75)$

$0.97(0.85-1.00)$

$0.82(0.60-0.95)$

$0.64(0.45-0.80)$

$0.74(0.55-0.88)$

$0.40(0.25-0.57)$

$0.76(0.55-0.91)$

$0.84(0.71-0.92)$

$0.74(0.56-0.87)$

$0.59(0.43-0.73)$

Pooled specificity $=0.67$ (0.64 to 0.71$)$

$\chi^{2}=87.21 ; \mathrm{df}=16(p=0.0000)$

Inconsistency $\left(I^{2}\right)=81.7 \%$

(b)

FIGURE 4: Summary sensitivity and specificity of PSR for high-risk varices in liver cirrhosis. (a) Summary sensitivity; (b) summary specificity. 
performance for varies. As previous studies have shown [5355], serum markers cannot be used to identify varices for the low-moderate diagnostic accuracy. Computer tomography has similar summary sensitivity (0.896) and specificity (0.723) compared to PSR [56]. In addition, the diagnostic accuracy of PSR for varices was slightly higher than spleen stiffness measurement [57] and liver stiffness measurement [58]. Their summary sensitivities were 0.78 and 0.87 , respectively. The summary specificities were 0.76 and 0.53 , respectively. While the diagnostic accuracy of PSR for varices was slightly lower than capsule endoscopy [59], its summary sensitivity and specificity were 0.85 and 0.84 , respectively. Some studies reported that splenoportal index and congestion index have high diagnostic accuracy. Their sensitivities and specificities were both greater than $80 \%$ [60, 61]. However, there are no systematic studies to evaluate their performance of varices.

Our study has some limitations. (1) 39 papers lacking relevant data were excluded, out of which some reported that PSR had no statistically significant difference in predicting the presence of varices. (2) Most analyses had significant heterogeneity. It may be attributed to the different selection criteria of patients, such as the prevalence of decompensated cirrhosis, etiologies of cirrhosis, and history of variceal bleeding. We have no evidence to support this assumption. The same situation was also found in the previous metaanalyses [2,3]. (3) The publication bias exists in the study. (4) Most TP, FP, FN, and TN were recalculated using sensitivities and specificities, which may introduce some errors. (5) PSR is not applicable to patients with a history of splenectomy.

In conclusion, PSR can be used to identify varices in liver cirrhosis. PSR had a high sensitivity in viral liver cirrhosis.

\section{Abbreviations}

PSR: Platelet count to spleen diameter ratio

HCC: Hepatocellular carcinoma

TP: $\quad$ True positive

FP: $\quad$ False positive

FN: $\quad$ False negative

TN: $\quad$ True negative

AUSROC: Area under the summary receiver operating characteristic curve

SE: $\quad$ Standard error

CI: $\quad$ Confidence interval

PLR: $\quad$ Positive likelihood ratio

NLR: $\quad$ Negative likelihood ratio

DOR: Diagnostic odds ratio.

\section{Competing Interests}

The authors declare that they have no competing interests.

\section{References}

[1] E. Giannini, F. Botta, P. Borro et al., "Platelet count/spleen diameter ratio: proposal and validation of a non-invasive parameter to predict the presence of oesophageal varices in patients with liver cirrhosis," Gut, vol. 52, no. 8, pp. 1200-1205, 2003.
[2] L. Ying, X. Lin, Z.-L. Xie, Y.-P. Hu, and K.-Q. Shi, "Performance of platelet count/spleen diameter ratio for diagnosis of esophageal varices in cirrhosis: a meta-analysis," Digestive Diseases and Sciences, vol. 57, no. 6, pp. 1672-1681, 2012.

[3] S. Chawla, A. Katz, B. M. Attar, A. Gupta, D. S. Sandhu, and R. Agarwal, "Platelet count/spleen diameter ratio to predict the presence of esophageal varices in patients with cirrhosis: a systematic review," European Journal of Gastroenterology and Hepatology, vol. 24, no. 4, pp. 431-436, 2012.

[4] P. F. Whiting, A. W. S. Rutjes, M. E. Westwood et al., "Quadas-2: a revised tool for the quality assessment of diagnostic accuracy studies," Annals of Internal Medicine, vol. 155, no. 8, pp. 529-536, 2011.

[5] X.-D. Xu, C.-F. Xu, J.-J. Dai, J.-Q. Qian, and X. Pin, "Ratio of platelet count/spleen diameter predicted the presence of esophageal varices in patients with schistosomiasis liver cirrhosis," European Journal of Gastroenterology \& Hepatology, vol. 28, no. 5, pp. 588-591, 2016.

[6] Y. Takuma, K. Nouso, Y. Morimoto et al., "Portal hypertension in patients with liver cirrhosis: diagnostic accuracy of spleen stiffness," Radiology, vol. 279, no. 2, pp. 609-619, 2016.

[7] T. Y. Kim, T. Y. Kim, Y. Kim, S. Lim, W. K. Jeong, and J. H. Sohn, "Diagnostic performance of shear wave elastography for predicting esophageal varices in patients with compensated liver cirrhosis," Journal of Ultrasound in Medicine, vol. 35, no. 7, pp. 1373-1381, 2016.

[8] A. Karatzas, C. Triantos, M. Kalafateli et al., "Multidetector computed tomography versus platelet/spleen diameter ratio as methods for the detection of gastroesophageal varices," Annals of Gastroenterology, vol. 29, no. 1, pp. 71-78, 2016.

[9] D. Zhao, H. Mao, C.-C. Huang, and S.-H. Zhang, "Study on noninvasive prediction indicators for incidence of esophageal varices of patients with liver cirrhosis caused by hepatitis B," Journal of Shanghai Jiaotong University, vol. 35, no. 3, pp. 386390, 2015.

[10] G. Valero and G. Olympia, "A retrospective study on noninvasive predictors of esophageal varices in patients with liver cirrhosis, a Makati Medical Center experience," Journal of Gastroenterology and Hepatology (Australia), vol. 30, article 358, 2015.

[11] H. Stefanescu, C. Radu, B. Procopet et al., "Non-invasive ménage à trois for the prediction of high-risk varices: stepwise algorithm using lok score, liver and spleen stiffness," Liver International, vol. 35, no. 2, pp. 317-325, 2015.

[12] E. J. Cho, M. Y. Kim, J.-H. Lee et al., "Diagnostic and prognostic values of noninvasive predictors of portal hypertension in patients with alcoholic cirrhosis," PLoS ONE, vol. 10, no. 7, Article ID e0133935, 2015.

[13] A. M. Albreedy, "Platelet count to spleen diameter ratio and to spleen area ratio as predictors for esopha-geal varices in chronic hepatitis C patients with liver cirrhosis," Journal of the Egyptian Society of Parasitology, vol. 45, no. 3, pp. 485-492, 2015.

[14] S. Zafar and M. M. Latif, "Diagnostic accuracy of platelet count/spleen diameter ratio for detection of esophageal varices in cirrhotic patients taking endoscopy as gold standard," Pakistan Journal of Medical and Health Sciences, vol. 8, no. 4, pp. 951-954, 2014.

[15] L. Wang, J. Hu, S. Dong et al., "Noninvasive prediction of large esophageal varices in liver cirrhosis patients," Clinical and Investigative Medicine, vol. 37, no. 1, pp. E38-E46, 2014.

[16] A. González-Ojeda, G. Cervantes-Guevara, M. Chávez-Sánchez et al., "Platelet count/spleen diameter ratio to predict 
esophageal varices in Mexican patients with hepatic cirrhosis," World Journal of Gastroenterology, vol. 20, no. 8, pp. 2079-2084, 2014.

[17] D. Chiodi, N. Hernández, G. Saona et al., "Noninvasive diagnosis of esophageal varices in cirrhotic patients," Acta Gastroenterologica Latinoamericana, vol. 44, no. 2, pp. 108-113, 2014.

[18] P. Sharma, V. Kirnake, P. Tyagi et al., "Spleen stiffness in patients with cirrhosis in predicting esophageal varices," American Journal of Gastroenterology, vol. 108, no. 7, pp. 1101-1107, 2013.

[19] Y. Saad, M. Said, M. O. Idris, A. Rabee, and Z. Salama, "Liver stiffness measurement by fibroscan predicts the presence and size of esophageal varices in egyptian patients with HCV related liver cirrhosis," Journal of Clinical and Diagnostic Research, vol. 7, no. 10, pp. 2253-2257, 2013.

[20] P. A. Rajendran, A. Philip, D. Krishnadas, K. C. George, Jayaprakash, and S. Devi, "Platelet count to spleen size ratioa simple noninvasive tool to identify small varices from large varices in patients having cirrohsis and portal hypertension," Journal of Clinical and Experimental Hepatology, vol. 3, no. 1, p. S93, 2013.

[21] A. R. Masjedizadeh, E. Hajiani, P. A. Nejad, J. Hashemi, A. A. Shayesteh, and Z. Yasin, "Efficacy platelet/spleen diameter ratio for detection of esophageal varices in cirrhotic patients," Journal of Gastroenterology and Hepatology Research, vol. 2, no. 5, pp. 590-592, 2013.

[22] V. Calvaruso, F. Bronte, E. Conte, F. Simone, A. Craxì, and V. Di Marco, "Modified spleen stiffness measurement by transient elastography is associated with presence of large oesophageal varices in patients with compensated hepatitis $C$ virus cirrhosis," Journal of Viral Hepatitis, vol. 20, no. 12, pp. 867-874, 2013.

[23] S. Nisar, S. Nazir, A. Butt, A. Hussain, and K. R. Yousaf, "Validity of platelet count/spleen diameter ratio for the noninvasive diagnosis of esophageal varices in cirrhotic patients," Pakistan Journal of Medical and Health Sciences, vol. 6, no. 1, pp. 269-274, 2012.

[24] M. Mangone, A. Moretti, F. Alivernini et al., "Platelet count/spleen diameter ratio for non-invasive diagnosis of oesophageal varices: is it useful in compensated cirrhosis?" Digestive and Liver Disease, vol. 44, no. 6, pp. 504-507, 2012.

[25] A. K. Mahassadi, F. Y. Bathaix, C. Assi et al., "Usefulness of noninvasive predictors of oesophageal varices in black african cirrhotic patients in Cte d'Ivoire (West Africa)," Gastroenterology Research and Practice, vol. 2012, Article ID 216390, 10 pages, 2012.

[26] A. Colecchia, L. Montrone, E. Scaioli et al., "Measurement of spleen stiffness to evaluate portal hypertension and the presence of esophageal varices in patients with HCV-related cirrhosis," Gastroenterology, vol. 143, no. 3, pp. 646-654, 2012.

[27] M. Al-Dahshan, "Clinical application of transient elastography in prediction of portal hypertension related complication in patients with chronic liver diseases," Journal of the Egyptian Society of Parasitology, vol. 42, no. 1, pp. 79-88, 2012.

[28] H. Stefanescu, M. Grigorescu, M. Lupsor, B. Procopet, A. Maniu, and R. Badea, "Spleen stiffness measurement using fibroscan for the noninvasive assessment of esophageal varices in liver cirrhosis patients," Journal of Gastroenterology and Hepatology (Australia), vol. 26, no. 1, pp. 164-170, 2011.

[29] J. R. Mosqueira, J. B. Montiel, D. Rodríguez, and E. Monge, "Evaluation of the diagnostic test of index platelet counts/size spleen, as a predictor of the presence of esophageal varices in cirrhosis," Revista de Gastroenterología del Perú, vol. 31, no. 1, pp. 11-16, 2011.
[30] S. Esmat and L. Rashid, "A comparative study between three noninvasive predictors of oesophageal varices in post hepatitis $\mathrm{C}$ virus liver cirrhosis in Egypt," Acta Gastro-Enterologica Belgica, vol. 74, no. 4, pp. 497-502, 2011.

[31] J. V. Cherian, N. Deepak, R. P. Ponnusamy, A. Somasundaram, and V. Jayanthi, "Non-invasive predictors of esophageal varices," Saudi Journal of Gastroenterology, vol. 17, no. 1, pp. 64-68, 2011.

[32] A. Agha, M. Abdulhadi, S. Marenco et al., "Use of the platelet count/spleen diameter ratio for the noninvasive diagnosis of esophageal varices in patients with schistosomiasis," Saudi Journal of Gastroenterology, vol. 17, no. 5, pp. 307-311, 2011.

[33] M. A. Abu El Makarem, M. E. Shatat, Y. Shaker et al., "Platelet count/bipolar spleen diameter ratio for the prediction of esophageal varices: the special Egyptian situation: noninvasive prediction of esophageal varices," Hepatitis Monthly, vol. 11, no. 4, pp. 278-284, 2011.

[34] E. Schwarzenberger, T. Meyer, V. Golla, N. P. Sahdala, and A. D. Min, "Utilization of platelet count spleen diameter ratio in predicting the presence of esophageal varices in patients with cirrhosis," Journal of Clinical Gastroenterology, vol. 44, no. 2, pp. 146-150, 2010.

[35] A. Sarangapani, C. Shanmugam, M. Kalyanasundaram, B. Rangachari, P. Thangavelu, and J. K. Subbarayan, "Noninvasive prediction of large esophageal varices in chronic liver disease patients," Saudi Journal of Gastroenterology, vol. 16, no. 1, pp. 3842, 2010.

[36] E. H. Nashaat, H. Abd-Elaziz, M. Sabry, and A. A. Ibrahim, "Non-endoscopic predictors of esophageal varices and portal hypertensive gastropathy," Nature and Science, vol. 8, no. 6, pp. 43-50, 2010.

[37] Â. Z. De Mattos, A. A. De Mattos, F. F. Vianna, M. I. Musskopf, J. C. Pereira-Lima, and A. C. Maciel, "Platelet count/spleen diameter ratio: analysis of its capacity as a predictor of the existence of esophageal varices," Arquivos de Gastroenterologia, vol. 47, no. 3, pp. 275-278, 2010.

[38] R. Barikbin, A. Hekmatnia, N. Omidifar, M. Farghadani, and P. Adibi, "Prediction severity of esophageal varices: a new cutoff point for Platelet count/spleen diameter ratio," Minerva Gastroenterologica e Dietologica, vol. 56, no. 1, pp. 1-6, 2010.

[39] M. A. Sharif, R. Firdous, T. A. Khan, T. H. Rizvi, M. Shani, and M. M. Elahi, "Platelet count/splenic size ratio: a parameter to predict the presence of oesophageal varices in cirrhotics," in Proceedings of the Asia Pacific Microwave Conference, vol. 3, pp. 23-26, 2009.

[40] C. Cammà, S. Petta, V. Di Marco et al., "Insulin resistance is a risk factor for esophageal varices in hepatitis C virus cirrhosis," Hepatology, vol. 49, no. 1, pp. 195-203, 2009.

[41] F. Barrera, A. Riquelme, A. Soza et al., "Platelet count/spleen diameter ratio for non-invasive prediction of high risk esophageal varices in cirrhotic patients," Annals of Hepatology, vol. 8, no. 4, pp. 325-330, 2009.

[42] A. Agha, E. Anwar, K. Bashir, V. Savarino, and E. G. Giannini, "External validation of the pPlatelet count/spleen diameter ratio for the diagnosis of esophageal varices in hepatitis $C$ virusrelated cirrhosis," Digestive Diseases and Sciences, vol. 54, no. 3, pp. 654-660, 2009.

[43] J. Y. Yu, X. N. Liu, D. Wang, and J. J. Cui, "Non-invasive predictive factors of esophageal varices in patients with posthepatitis B cirrhosis," Journal of Binzhou Medical University, vol. 31, no. 5, pp. 345-349, 2008 (Chinese).

[44] W. Tao, S. Q. Yang, X. C. Lv, and L. Yang, "Value of the platelet count/spleen diameter ratio on diagnosis of esophageal varices 
in patients with cirrhosis," Journal of Ningxia Medical College, vol. 30, no. 3, pp. 349-350, 2008 (Chinese).

[45] A. Parrino, V. Di Gesaro, A. Terranova et al., "Non-invasive diagnosis of esophageal varices in cirrhotic patients: endoscopic vs ultrasonographic findings," Acta Medica Mediterranea, vol. 24, no. 1, pp. 11-18, 2008.

[46] W. W. Baig, M. V. Nagaraja, M. Varma, and R. Prabhu, "Platelet count to spleen diameter ratio for the diagnosis of esophageal varices: is it feasible?" Canadian Journal of Gastroenterology, vol. 22, no. 10, pp. 825-828, 2008.

[47] J. B. Lei and T. Z. Huang, "Value of the platelet count/spleen diameter ratio on predicting esophageal varices of liver cirrhosis," Practical Clinical Medicine, vol. 8, no. 10, pp. 25-27, 2007 (Chinese).

[48] G. H. Sethar, R. Ahmed, S. K. Rathi, and N. A. Shaikh, "Platelet count/splenic size ratio: a parameter to predict the presence of esophageal varices in cirrhotics," Journal of the College of Physicians and Surgeons Pakistan, vol. 16, no. 3, pp. 183-186, 2006.

[49] G. M. Legasto, J. Sevilla, A. Balay et al., "Platelet count/spleen diameter ratio: a noninvasive parameter to predict the presence of esophageal varices," Phillippine Journal of Gastroenterology, vol. 2, pp. 33-38, 2006.

[50] E. G. Giannini, A. Zaman, A. Kreil et al., "Platelet count/spleen diameter ratio for the noninvasive diagnosis of esophageal varices: results of a multicenter, prospective, validation study," American Journal of Gastroenterology, vol. 101, no. 11, pp. 25112519, 2006.

[51] S. Pleština, R. Pulanić, M. Kralik, S. Pleština, and M. Samaržija, "Color Doppler ultrasonography is reliable in assessing the risk of esophageal variceal bleeding in patients with liver cirrhosis," Wiener Klinische Wochenschrift, vol. 117, no. 19-20, pp. 711-717, 2005.

[52] E. G. Giannini, F. Botta, P. Borro et al., "Application of the platelet count/spleen diameter ratio to rule out the presence of oesophageal varices in patients with cirrhosis: a validation study based on follow-up," Digestive and Liver Disease, vol. 37, no. 10, pp. 779-785, 2005.

[53] X. Qi, H. Li, J. Chen et al., "Serum liver fibrosis markers for predicting the presence of gastroesophageal varices in liver cirrhosis: a retrospective cross-sectional study," Gastroenterology Research and Practice, vol. 2015, Article ID 274534, 6 pages, 2015.

[54] H. Deng, X. Qi, Y. Peng et al., "Diagnostic accuracy of APRI, AAR, FIB-4, FI, and king scores for diagnosis of esophageal varices in liver cirrhosis: A Retrospective Study," Medical Science Monitor, vol. 21, pp. 3961-3977, 2015.

[55] H. Deng, X. Qi, and X. Guo, "Diagnostic accuracy of APRI, AAR, FIB-4, FI, king, lok, forns, and fibroindex scores in predicting the presence of esophageal varices in liver cirrhosis: a systematic review and meta-analysis," Medicine, vol. 94, no. 42, Article ID e1795, 2015.

[56] Y.-J. Tseng, X.-Q. Zeng, J. Chen, N. Li, P.-J. Xu, and S.-Y. Chen, "Computed tomography in evaluating gastroesophageal varices in patients with portal hypertension: a meta-analysis," Digestive and Liver Disease, vol. 48, no. 7, pp. 695-702, 2016.

[57] S. Singh, J. E. Eaton, M. H. Murad, H. Tanaka, H. Iijima, and J. A. Talwalkar, "Accuracy of spleen stiffness measurement in detection of esophageal varices in patients with chronic liver disease: systematic review and meta-analysis," Clinical Gastroenterology and Hepatology, vol. 12, no. 6, pp. 935-945.e4, 2014.
[58] K.-Q. Shi, Y.-C. Fan, Z.-Z. Pan et al., “Transient elastography: a meta-analysis of diagnostic accuracy in evaluation of portal hypertension in chronic liver disease," Liver International, vol. 33, no. 1, pp. 62-71, 2013.

[59] A. Colli, J. C. R. Gana, D. Turner et al., "Capsule endoscopy for the diagnosis of oesophageal varices in people with chronic liver disease or portal vein thrombosis," The Cochrane database of systematic reviews, vol. 10, Article ID CD008760, 2014.

[60] R. Chakrabarti, D. Sen, and V. Khanna, "Is non-invasive diagnosis of esophageal varices in patients with compensated hepatic cirrhosis possible by duplex Doppler ultrasonography?" Indian Journal of Gastroenterology, vol. 35, no. 1, pp. 60-66, 2016.

[61] H. S. Mahmoud, E. F. Mostafa, and M. A. W. Mohammed, "Role of portal haemodynamic parameters in prediction of oesophageal varices in cirrhotic patients," Arab Journal of Gastroenterology, vol. 15, no. 3-4, pp. 130-134, 2014. 


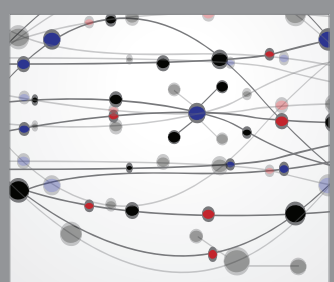

The Scientific World Journal
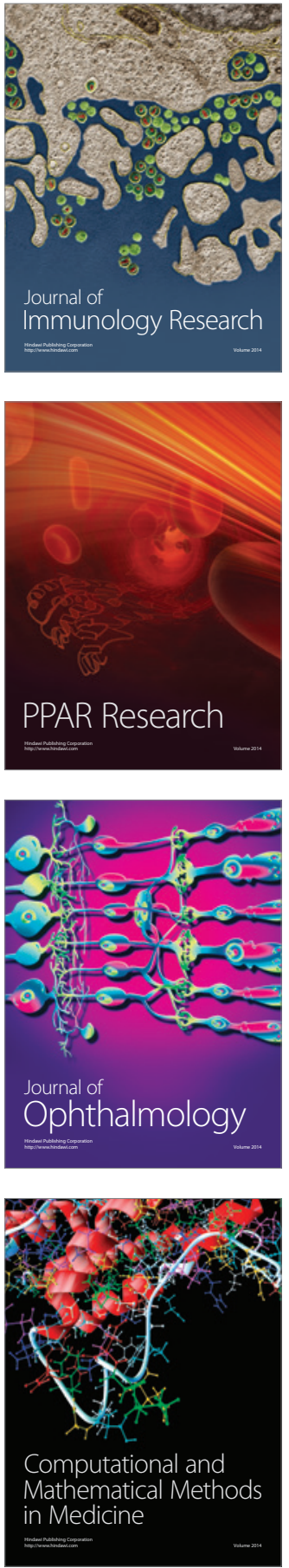

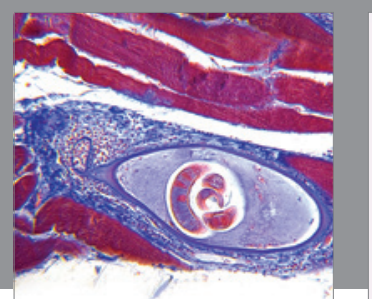

Gastroenterology Research and Practice
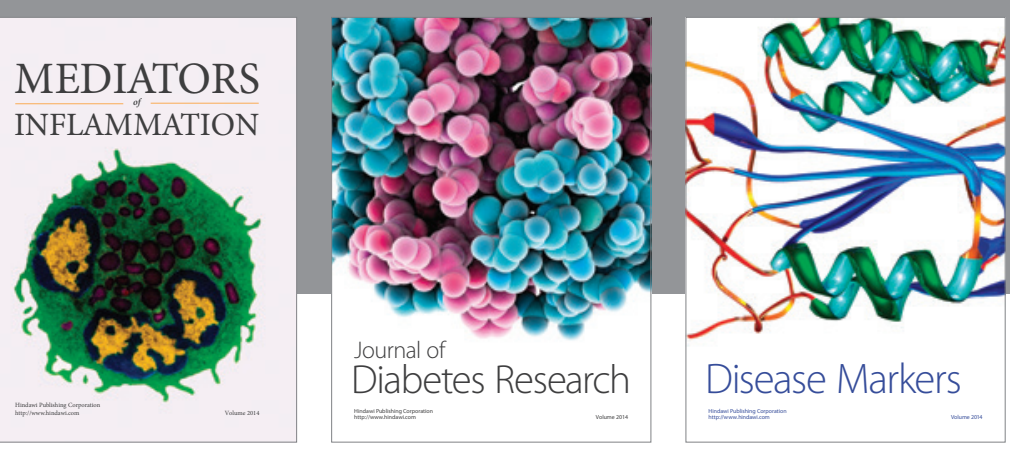

Disease Markers

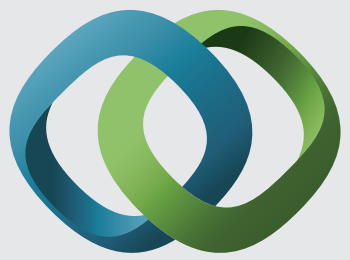

\section{Hindawi}

Submit your manuscripts at

https://www.hindawi.com
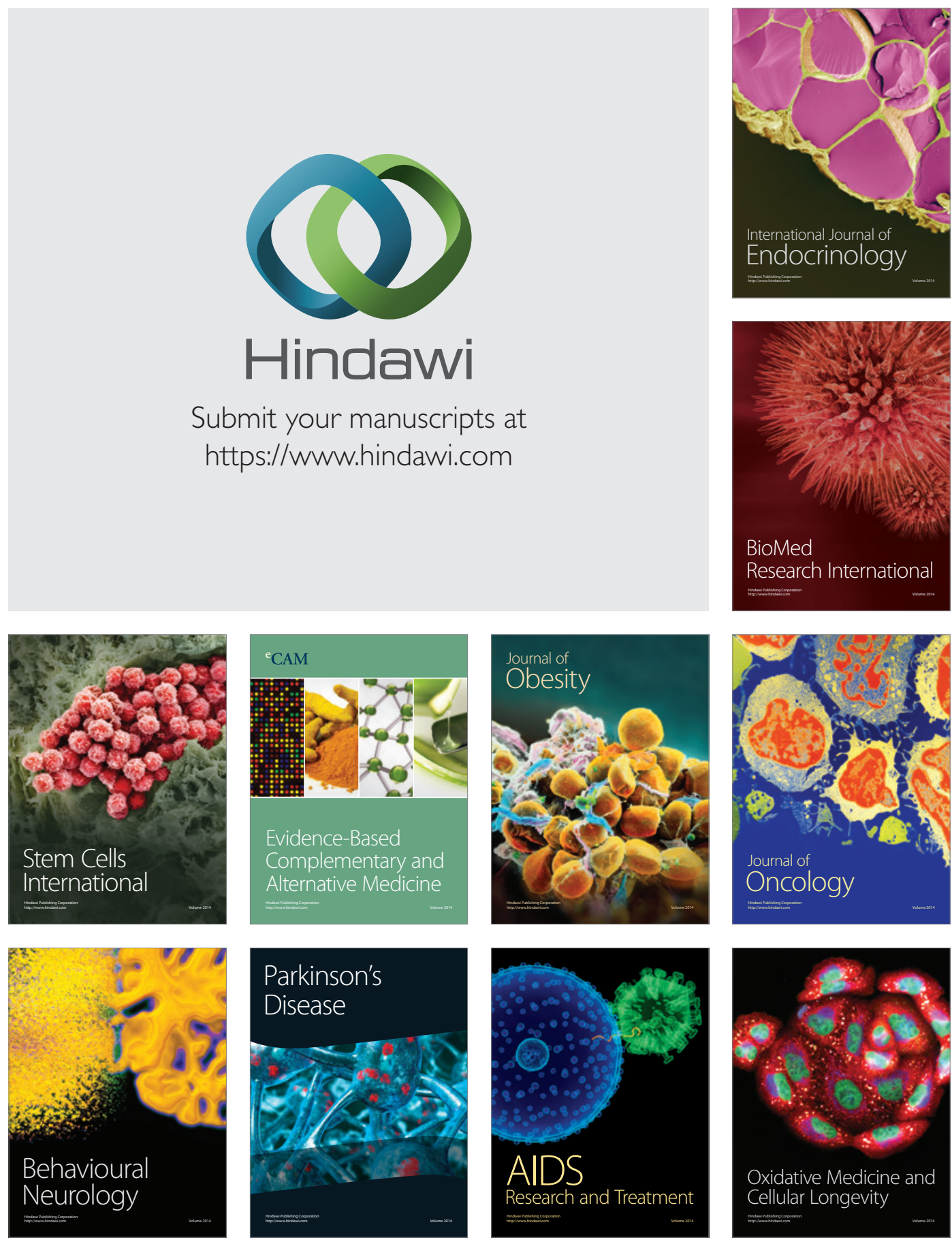